\title{
Quadruplex folding promotes the condensation of linker histones and DNAs via liquid-liquid phase separation
}

\author{
Masahiro Mimura†',, Shunsuke Tomita*,,, Yoichi Shinkai*, Takuya Hosokai**, Hiroyuki Kumeta//, \\ Tomohide Saiođ, Kentaro Shiraki†, Ryoji Kurita ${ }^{*}+, \neq, \S$
}

†Faculty of Pure and Applied Sciences, University of Tsukuba, 1-1-1 Tennodai, Tsukuba, Ibaraki 305-8573, Japan, *Health and Medical Research Institute, National Institute of Advanced Industrial Science \& Technology (AIST), 1-1-1 Higashi, Tsukuba, Ibaraki 305-8566, Japan, *Bomedical Research Institute, AIST, 1-1-1 Higashi, Tsukuba, Ibaraki 3058566, Japan, **National Metrology Institute, AIST, 1-1-1 Higashi, Tsukuba, Ibaraki 305-8565, Japan, //Faculty of Advanced Life Science, Hokkaido University, Sapporo, Hokkaido, 060-0810, Japan, $\uparrow$ Graduate School of Chemical Sciences and Engineering, Hokkaido University, Sapporo, Hokkaido, 060-8628, Japan, §DAILAB, DBT-AIST International Center for Translational and Environmental Research (DAICENTER), AIST, 1-1-1 Higashi, Tsukuba, Ibaraki, 305-8565, Japan, ${ }^{*}$ Corresponding author

KEYWORDS, Chromatin condensation, G-quadruplex, Histone, Liquid-liquid phase separation

\begin{abstract}
Liquid-liquid phase separation (LLPS) of proteins and DNA has recently emerged as a possible mechanism underlying the dynamic organization of chromatin. We herein report the role of DNA quadruplex folding in liquid droplet formation via LLPS induced by interactions between DNA and linker histone H1 (H1), a key regulator of chromatin organization. Fluidity measurements inside the droplets, binding assays using G-quadruplex-selective probes, and structural analyses based on circular dichroism demonstrated that quadruplex DNA structures, such as the G-quadruplex and i-motif, promote droplet formation with $\mathrm{H} 1$ and decrease molecular motility within droplets. The dissolution of the droplets in the presence of additives and the LLPS of the DNA structural units indicated that in addition to electrostatic interactions between the DNA and the intrinsically disordered region of $\mathrm{H} 1, \pi-\pi$ stacking between quadruplex DNAs could potentially drive droplet formation, unlike in the electrostatically driven LLPS of duplex DNA and H1. According to phase diagrams of anionic molecules with various conformations, the high LLPS ability associated with quadruplex folding arises from the formation of interfaces consisting of organized planes of guanine bases and the side surfaces with high charge density. Given that DNA quadruplex structures are well documented in heterochromatin regions, it is imperative to understand the role of DNA quadruplex folding in the context of intranuclear LLPS.
\end{abstract}

\section{${ }_{28}$ Introduction}

${ }_{29}$ Genomic DNA in eukaryotic cells wraps around histone pro30 tein cores to form nucleosomes, which are further com${ }_{31}$ pacted into chromatin. ${ }^{1}$ The level of chromatin condensa32 tion is closely related to gene transcription; ${ }^{2}$ heterochroma${ }_{3}$ tin is a tightly packed form that is inaccessible to polymer${ }_{34}$ ases and thus inactivates gene transcription, whereas gene 35 transcription is activated in euchromatin, in which the nu${ }_{36}$ cleosomes are loosely packed. ${ }^{3}$ Chromatin undergoes highly 37 dynamic changes in its condensed structure during a cell cy38 cle. However, the mechanisms that govern the organization 39 of chromatin remain largely unknown.

40 Liquid-liquid phase separation (LLPS) has emerged as a ${ }_{41}$ possible mechanism for the control of chromatin
42 organization through the promotion of nucleosome pack${ }_{43}$ ing. ${ }^{4}$ Biological LLPS is a process in which solutions of biom44 acromolecules spontaneously separate into two phases. ${ }^{5,6}$ 45 In such events, one phase is usually a small-volume droplet46 like phase in which the biomacromolecules are concen47 trated in aqueous media, while the other is the surrounding ${ }_{48}$ phase, which is depleted of the biomacromolecules. ${ }^{7}$ Multi49 valent weak intermolecular interactions involving intrinsi50 cally disordered regions (IDRs) of proteins, such as electro${ }_{51}$ static, cation- $\pi$, and $\pi-\pi$ interactions, play crucial roles in 52 LLPS. ${ }^{5,6}$ For instance, binding of the IDR-containing hetero53 chromatin protein HP $1 \alpha$ to the histone H3K9 methylation ${ }_{54}$ site induces LLPS in specific domains of heterochromatin. ${ }^{8,9}$ 55 LLPS also occurs in euchromatin regions that are rich in 56 acetylated histone tails when the transcriptional regulator 57 protein BRD4, which contains a long IDR, is co-localized. ${ }^{10,11}$ 
${ }_{58}$ Thus, the nature of the relationship between LLPS59 mediated chromatin-condensation and proteins is gradu60 ally determined.

Several reports have indicated that DNA is also involved 62 in the LLPS associated with chromatin condensation. The ${ }_{63}$ length of the inter-nucleosome linker DNA strongly affects 64 the LLPS of nucleosome arrays. ${ }^{10}$ Double-stranded DNA in65 duces LLPS in the presence of histone $\mathrm{H} 1,{ }^{12}$ which is capable 66 of regulating chromatin organization via binding to inter${ }_{67}$ nucleosome linker DNA. However, knowledge regarding the 68 structure of the DNA involved in LLPS-mediated chromatin69 condensation is still very limited. Therefore, in this work, 70 we have focused on the secondary structures of DNA, espe7 cially the most common G-quadruplex structure. ${ }^{13}$

72 G-quadruplex is a stacking planar structure formed 73 through Hoogsteen hydrogen bonds between four guanine ${ }_{4}$ residues (Figure $\left.1 \mathrm{~A}\right) .{ }^{13}$ Guanine-rich sequences with the po${ }_{5}$ tential to fold into the G-quadruplex structure are fre${ }_{6}$ quently observed in oncogene promoter sequences and te77 lomere regions, which are known as quadruplex clusters or ${ }_{8}$ G4 clusters, ${ }^{14,15}$ where they may promote or inhibit the ac9 cess of transcriptional factors or telomere binding proteins. ${ }^{16,17}$ Recently, it has been revealed that G-quadruplex sequences are also abundant in heterochromatin region; ${ }^{18}$ 2 however, the role of these sequences on chromatin conden${ }_{3}$ sation has not been clarified. Thus, we attempted to eluci${ }_{4}$ date the relationship between the G-quadruplex DNA struc5 ture and the LLPS of chromatin constituents using a variety 6 of sequences that are capable of forming quadruplex struc87 tures. The presented findings will facilitate the understand8 ing of the role of G-quadruplex structures in the cell nucleus 89 and chromatin condensation.

\section{${ }_{90}$ Results and Discussion}

\section{${ }_{91}$ LLPS of G-quadruplex-forming sSDNA with H1}

92 To investigate the effect of G-quadruplex formation on LLPS ${ }_{93}$ in the context of chromatin condensation, we chose histone ${ }_{94} \mathrm{H} 1$ (H1) and various single-stranded DNA (ssDNA) 95 sequences. $\mathrm{H} 1$ controls the packing density of nucleosomes 96 via non-specific electrostatic interactions between its ${ }_{97}$ positively charged lysine-rich IDR at its C-terminal and 98 negatively charged DNA (Figure 1B). ${ }^{19}$ Shakya et al. recently 99 reported that among histone proteins, H1 has the highest 100 ability to form droplets with DNA via LLPS. ${ }^{20}$ Four $22 \mathrm{nt}$ ${ }_{101}$ SsDNA sequences were initially prepared (Figure 1C): an 102 oncogene $c$-myc promoter sequence that can fold into a par103 allel G-quadruplex structure (Pu22) ${ }^{21}$; sequences in which 104 one or two of the successive guanines of Pu22 were re05 placed with adenine (Pu22-1 and Pu22-2, respectively); and 106 a simple repeat of deoxyadenylic acid (poly(dA)) with a ran107 dom coil structure. The nucleotides are arranged an all-anti 108 configuration in the parallel forms of G-quadruplexes, while 109 the antiparallel forms contain nucleotides in both syn and 110 anti configurations (Figures $1 \mathrm{~A}$ and S1A). The secondary ${ }_{111}$ structure of each ssDNA was examined by circular
112 dichroism (CD) measurements, which revealed that with 113 decreasing number of guanines, the content of G-quadru114 plex structures decreased (Pu22 to Pu22-1), whereby Pu221152 exhibits a random coil-like structure (for details, see sec116 tion 3 of the Supporting Information).

117 The turbidity of the aqueous solutions of the guanine118 containing sequences (Pu22, Pu22-1, and Pu22-2) in119 creased upon addition of $\mathrm{H} 1$ up to a certain concentration $120([\mathrm{H} 1] /[\mathrm{ssDNA}]=0.2-0.4)$ (Figure $2 \mathrm{~A})$, indicating that inter121 actions between the ssDNA sequences and $\mathrm{H} 1$ resulted in 122 the formation of large assemblies. Interestingly, the maxi123 mum turbidity values of the solutions increased with the 124 number of continuous guanines in the ssDNA sequence 125 (Pu22 > Pu22-1 > Pu22-2). The decreased turbidity at high 126 concentrations of $\mathrm{H} 1$ probably results from repulsive forces 127 that arise from the excess of positive charge; 12,23 thus, elec128 trostatic interactions between the cationic $\mathrm{H} 1$ and anionic 129 SSDNA are presumably a dominant force in the formation of 130 the assemblies. However, although poly $(\mathrm{dA})$, like the other ${ }_{131}$ SsDNA sequences, is anionic, the poly (dA) solution did not 132 exhibit an apparent increase in turbidity upon addition of ${ }_{133} \mathrm{H} 1$.

(A)

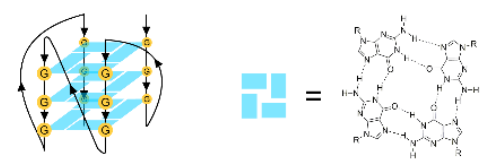

Parallel G-quadruplex G-quartet

(B)

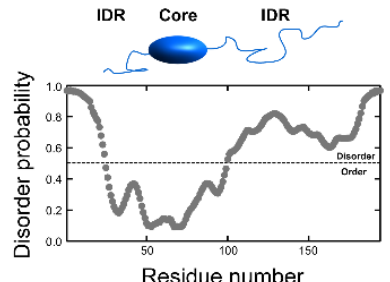

MTENSTSTPA AKPKRAKASK KSTDHPKYSD MIVAAIQAEK NRAGSSRQS QKYIKSHYKV GENADSQIKL SIKRLVTTGV LKQTKGVGAS GSFRLAKSDE PKRSVAFKKT KKEVKKVATP KKAAKPKKAA SKAPSKKPKA TPVKKAKKK AATPKKTKKP KTVKAKPVKA SKPKKTKPVK PKAKSSAKRT GKKK

(C)

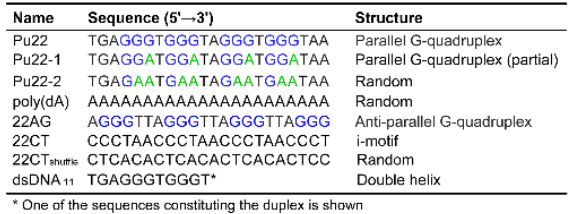

134

135 Figure 1. Sequence and higher order structure of the molecules 136 used in this study. (A) Schematic illustration of the parallel G${ }_{137}$ quadruplex DNA structure (left), which consists of stacked G138 quartets (right). (B) Disorder probability of the H1 structure as 139 predicted using the Protein DisOrder prediction System 140 (PrDOS). ${ }^{22}$ Regions of the sequence that exhibit a score bigger 141 than 0.5 are defined as intrinsically disordered regions (high142 lighted in light blue). The sequence of $\mathrm{H} 1$ from bovine thymus 143 was obtained from Uniprot code Q0IIJ2. (C) Sequences and ${ }_{144}$ structures of the ssDNA used in this study. 
(A)

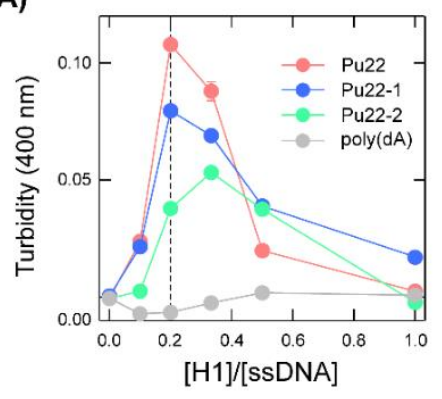

(D)

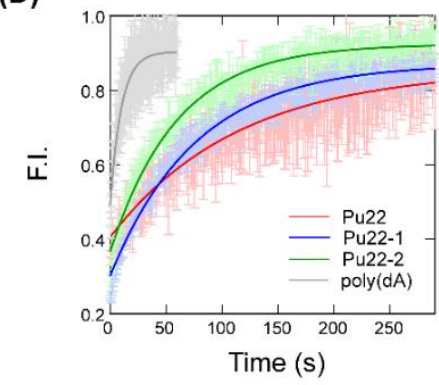

(B)

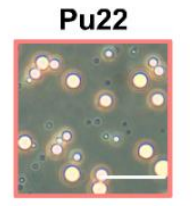

Pu22-2
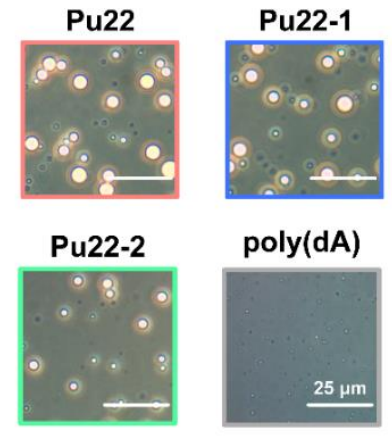

poly(dA)
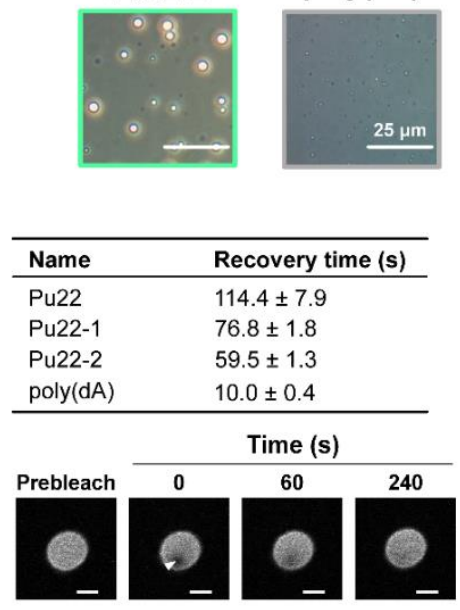

(C)

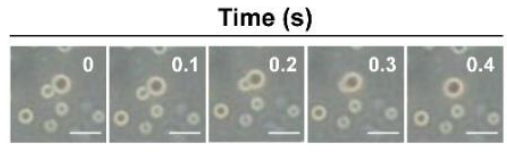

(E)
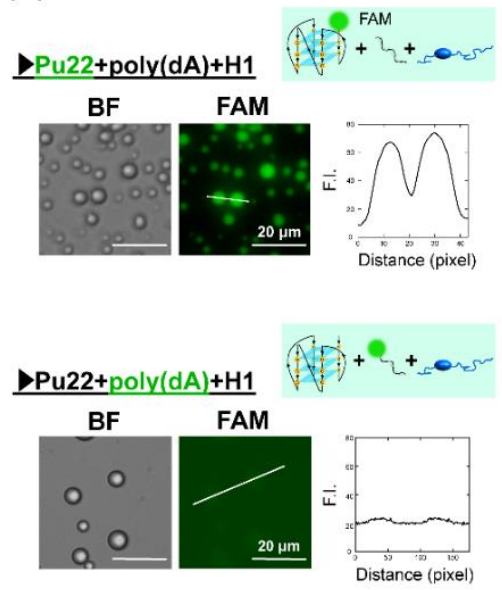

Figure 2. Liquid-like droplets of G-quadruplex-forming ssDNA with H1. (A) Turbidity of solutions that contain various ssDNA sequences $(10 \mu \mathrm{M})$ and $\mathrm{H} 1(0-10 \mu \mathrm{M})$. (B) Phase-contrast-microscopy images of solutions that contain ssDNA $(10 \mu \mathrm{M})$ and $\mathrm{H} 1(2 \mu \mathrm{M})$; scale bar $=25 \mu \mathrm{m}$. (C) Fusion process of the Pu22/H1 droplets; scale bar $=10 \mu \mathrm{m}$. (D) Left: FRAP recovery curves for the different ssDNA structures. Right: recovery times of fluorescence calculated by exponential fitting (colored lines; $\mathrm{N}=3$ ), and fluorescence images obtained during the FRAP measurement of a Pu22/H1 liquid droplet. The white arrowhead indicates the bleaching site; scale bar $=5 \mu \mathrm{m}$. (E) Selectivity of the ssDNA sequences for the droplet formation with H1. Either Pu22 or poly(dA) was modified with FAM, and both $(10 \mu \mathrm{M})$ were mixed with $\mathrm{H} 1(2 \mu \mathrm{M})$. The fluorescence intensity along the white line was quantified from the brightness of each pixel; scale bar $=20 \mu \mathrm{m}$. All experiments were carried out in $10 \mathrm{mM}$ Tris-EDTA buffer $(\mathrm{pH}=7.4)$.

154 Spherical assemblies were observed via phase-contrast 155 microscopy for all turbid ssDNA solutions $([\mathrm{H} 1] /[$ ssDNA $]=$ 156 0.2; Figure 2B), similarly to our recent studies of cationic 57 protein/anionic polymer pairs. ${ }^{24,25}$ Time-lapse images 158 showed rapid, sub-millisecond fusion of the assemblies 159 (Figure 2C). This behavior indicates that these assemblies 160 are not gel-like aggregates, but instead liquid-like droplets ${ }_{161}$ with highly fluid properties, as have been observed for 62 other phase-separating proteins. ${ }^{26}$ Consistent with the tur63 bidity measurements (Figure 2A), the size of the observed ${ }_{64}$ droplets decreased with decreasing number of continuous 65 guanines in the ssDNA sequence. In the case of poly(dA), 166 only small droplets (diameter $<1.0 \mu \mathrm{m}$ ) were formed. Simi${ }_{67}$ lar behavior was observed for other ssDNA sequences, in68 cluding an anti-parallel G-quadruplex sequence present in 169 telomeric regions (22AG), its derivatives, and a simple re170 peat of deoxythymidylic acid (poly(dT)) (Figure S1). Thus, ${ }_{171}$ SsDNA sequences capable of forming G-quadruplex are 172 likely to have a high ability to form droplets with H1.

The effect of the ssDNA sequence on the fluidity inside 74 the droplet was compared using fluorescence recovery after 75 photobleaching (FRAP), which is a common method for 176 evaluating the motility of molecules inside the droplets. ${ }^{27}$ 177 The diffusion rate of the carboxyfluorescein (FAM)-

178 modified ssDNA sequences increased with decreasing num179 ber of continuous guanines [poly(dA) > Pu22-2 > Pu22-1 > $\left.{ }_{180} \mathrm{Pu} 22\right]$, i.e., in the opposite order of the content of quadru181 plex structures (Figure 2D). Interestingly, the density of the 182 SsDNAs inside the droplet was correlated with their motility 183 (Figure S2), i.e., the higher the G-quadruplex content, the 184 lower the density inside the droplet, despite the stronger in185 termolecular interactions inside the droplet. Therefore, the ${ }_{186}$ G-quadruplex folding also controls the motility and density 187 of the molecules inside the resulting droplets, which could 188 potentially affect cellular functions such as the inhibition of 189 gene transcription.

190 To study the sequence selectivity of the droplet for191 mation, either Pu22 or poly(dA) was labeled with FAM, and 192 then both were mixed with H1. When Pu22 was labeled, the 193 inside of the droplet emitted strong fluorescence, whereas 194 the fluorescence inside and outside of the droplet was com195 parable for labeled poly(dA) (Figure 2E), suggesting se196 quence selectivity not only in the formation of droplets with ${ }_{197} \mathrm{H} 1$, but also in the incorporation of ssDNA into the resulting 198 droplets.

199 Based on these results, it seems feasible to conclude that 200 SsDNA sequences that can fold into G-quadruplex structures 201 plays a significant role in (i) the generation of LLPS through 
(A)
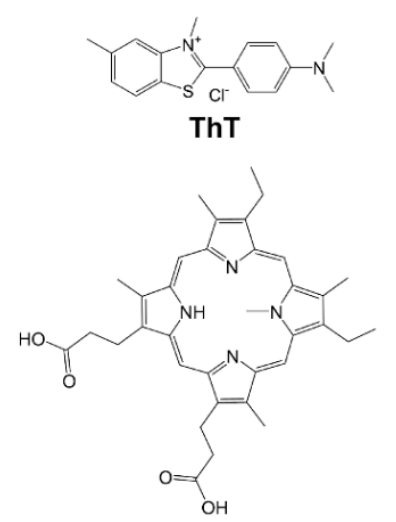

NMM

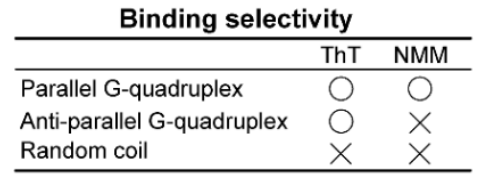

(B)

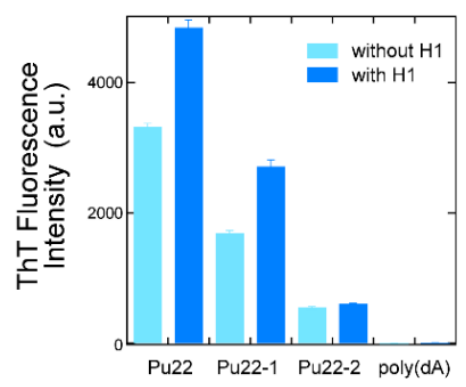

(D)

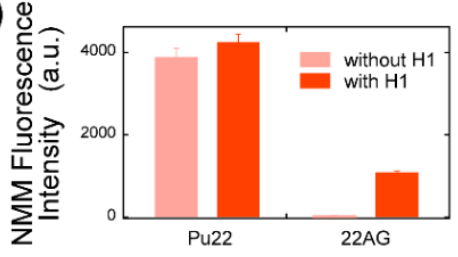

(C)

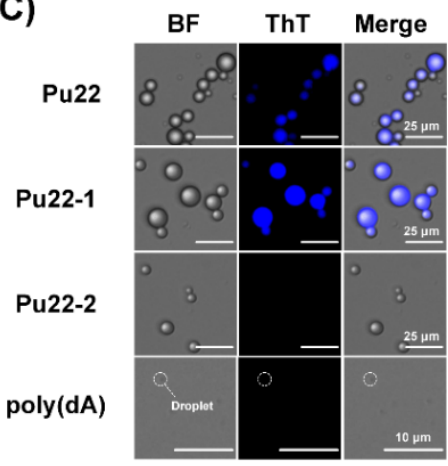

(E)

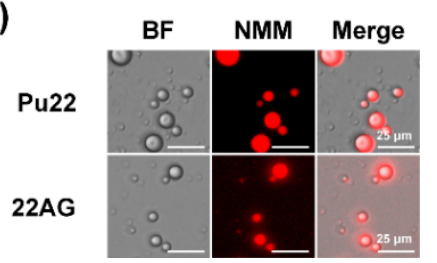

Figure 3. Analysis of G-quadruplex structures inside the liquid droplets using fluorescent molecular probes. (A) Chemical structures of the fluorescent probes ThT and NMM, which exhibit greatly enhanced fluorescence upon their selective binding to G-quadruplex structures. The binding selectivity of ThT and NMM is taken from refs ${ }^{28}$ and ${ }^{29}$, respectively. Fluorescence intensity of (B) ThT and (D) NMM in solutions that contain ssDNA $(10 \mu \mathrm{M})$ with or without $\mathrm{H} 1(2 \mu \mathrm{M})$. Fluorescence microscopy images of ssDNA/H1 liquid droplets after the addition of (C) ThT and (E) NMM; scale bar $=25 \mu \mathrm{m}(10 \mu \mathrm{m}$ for poly(dA)).

208 interactions with H1, (ii) the motility and density of the mol209 ecules inside the formed droplets, and (iii) the ability of the 210 droplets to incorporate other ssDNAs.

\section{${ }_{211}$ G-quadruplex folding within liquid-like droplets}

212 The stability of the G-quadruplex structure is affected by ${ }_{213}$ protein binding. ${ }^{17}$ Since the components of the droplets 214 formed through LLPS are generally concentrated within the 215 droplets by a factor of several to several hundred compared 216 to the surrounding phase, ${ }^{10,30}$ the G-quadruplex content 217 might fluctuate due to the presumably high concentration 218 of $\mathrm{H} 1$ inside the droplets. Therefore, we investigated the 219 folding state of the ssDNA within the droplet using two 220 fluorogenic probes that selectively bind to G-quadruplex 221 structures, thioflavin T (ThT) and $N$-methylmesoporphyrin 222 IX (NMM) (Figure 3A). ThT binds to G-quadruplex 223 structures regardless of their configration, ${ }^{28}$ while NMM 224 recognizes only parallel-folded G-quadruplexes. ${ }^{29}$ These 225 probes are almost nonfluorescent in aqueous solution, but 226 exhibit strong emission when bound to G-quadruplex 227 structures.

In the absence of $\mathrm{H} 1$, the overall fluorescence intensity of 29 the solutions in the presence of ThT follows the order Pu22 $230>\mathrm{Pu} 22-1>\mathrm{Pu} 22-2>>$ Poly(dA), i.e., the abundance of G231 quadruplex structures increases with increasing number of 232 successive guanines in the ssDNA sequence (Figure 3B; for 233 the fluorescence spectra, see Figure S3A). The fluorescence 234 intensity of ThT after the droplet formation is comparable 235 for the $\mathrm{Pu} 22-2 / \mathrm{H} 1$ or poly $(\mathrm{dA}) / \mathrm{H} 1$ solutions, while that of 236 the $\mathrm{Pu} 22 / \mathrm{H} 1$ and $\mathrm{Pu} 22-1 / \mathrm{H} 1$ solutions increases $1.5-$ and
237 1.6-fold, respectively (Figure 3B). These fluorescence en238 hancements were not due to changes in the dielectric con239 stant within the droplets, peak shifts, or changes in the flu240 orescence lifetimes, but instead to the enhanced formation 241 of the G-quadruplex structure to which ThT can bind (for 242 details, see section 4 of the Supporting Information).

243 Fluorescence microscopy images of the Pu22 and Pu22-1 244 solutions showed significant fluorescence inside the drop245 lets (Figure 3C), demonstrating that the binding of ThT to 246 the G-quadruplex occurred mainly inside the droplets. In 247 contrast, the Pu22-2 and poly(dA) solutions exhibited very 248 weak fluorescence. We found that other fluorescent dyes (8249 anilino-1-naphthalenesulfonic acid, Nile red, and rhoda250 mine 6G) tended to be concentrated in the ssDNA/H1 drop251 lets, regardless of the dye structure or ssDNA sequence (Fig252 ure S4). Unlike these dyes, in addition to being concentrated 253 in the ssDNA-rich droplets, ThT must be bound to the G254 quadruplex in order to emit visible fluorescence, as indi255 cated in section 4 of the Supporting Information.

The CD spectra of the droplet suspensions and con7 densed phases supported the possibility of the promotion 258 and maintenance of G-quadruplex folding inside the drop259 lets (for details, see section 5 of the Supporting Infor260 mation). The measurements of the condensed phase using 261 high-resolution magic angle spinning nuclear magnetic res262 onance (HR-MAS-NMR), which is a suitable technique for 263 the NMR measurement of samples with high viscosity, ${ }^{31}$ 264 suggested that the conformation of quadruplex Pu22 inside 265 the droplets may be polymorphic within the range to which 
${ }_{266}$ G-quadruplex specific probes can bind (for details, see sec267 tion 5 of the Supporting Information).

The origin of the enhancement of the G-quadruplex fold29 ing was explored using two model cationic polymers in270 stead of H1: a simple repeat poly-L-lysine (PLL), and PLL 271 with a polyethylene glycol chain, which inhibits droplet 272 growth (for details, see section 6 of the Supporting Infor273 mation). Briefly, the results suggest that one of the domi274 nant factors was the direct non-specific interactions be275 tween polycationic chains and ssDNAs. Such interactions 276 could potentially lead to suppression of the inter- and intra77 molecular repulsion of ssDNAs by neutralization of the 278 phosphate groups and subsequent promotion of nucleobase 279 stacking. Another possibility is that the molecular crowding 280 causes dehydration of G-quadruplex structures, which sta281 bilizes this conformation. ${ }^{32}$ The dense, crowded environ282 ment inside the droplets may also lead to enhanced folding.

283 In the case of a telomere-derived sequence (22AG) that is 284 capable of folding into an anti-parallel G-quadruplex struc285 ture, ThT gave results similar to Pu22 (Figure S3B), whereas 86 NMM exhibited interesting behavior. Aqueous solutions of $287 \mathrm{Pu} 22$ showed similar strong fluorescence with or without ${ }_{288} \mathrm{H} 1$ after the addition of NMM, which is specific to parallel89 type G-quadruplexes (Figure 3D), indicating that the paral290 lel G-quadruplex structure of Pu22 is not significantly dena291 tured by H1. On the other hand, substantial NMM emission 292 was observed from the entire solution of anti-parallel 22AG 93 only after droplets were formed by the addition of H1. Sim294 ilar to the emission of ThT, that of NMM was concentrated 295 inside the droplets for both ssDNA sequences (Figure 3E). 296 The enhancement in the fluorescence after droplet for297 mation for 22AG suggests that the G-quadruplex structures 298 of 22AG transitioned from the anti-parallel to the parallel 299 form. The CD spectra of the condensed phases also indicate 300 the possibility of the structural transition (Figure S18B). 301 Similar to the enhanced folding of Pu22, this structural tran302 sition seems to be mainly due to the interaction with the cat303 ionic tail of H1 (for details, see section 6 of the Supporting 304 Information), but the molecularly crowded environments 305 might also be significant. ${ }^{33}$ It has been reported that the 306 anti-parallel to parallel transition in telomere-derived ${ }_{307}$ SsDNA inhibits telomerase processability. ${ }^{33}$ Thus, if transi308 tion of the G-quadruplex structures of 22AG is coupled to 309 the droplet-formation-inducing interaction with $\mathrm{H} 1$, it was 310 presumably involved in the telomere activity switching 11 mechanism in the cell nucleus.

\section{Generality of the promotion of LLPS by the quadruplex conformation}

314 To clarify the generality of the promotion of LLPS by quad15 ruplex structures in ssDNA, we tested another quadruplex ${ }_{316}$ structure formed by successive cytosine bases, the so-called 317 i-motif (Figure 4A). ${ }^{34}$ As in the case of G-quadruplex, the in318 crease in turbidity upon addition of $\mathrm{H} 1$ was greater for an i319 motif-forming sequence (22CT) than for a shuffled variant 20 of this sequence in which the cytosines are not successive
${ }_{321}\left(22 \mathrm{CT}_{\text {shuffle }}\right)$ (Figure 4B). Consistent with the turbidity re322 sults, the droplets formed by 22CT were significantly larger 323 than those formed by $22 \mathrm{CT}_{\text {shuffle }}$ (Figure $4 \mathrm{~B}$ ). The CD spec324 trum of the condensed phase suggested that the folding of $32522 \mathrm{CT}$ into the i-motif was promoted by the process of drop326 let formation (Figure S18C).

327 Thus, the dependence of the phase separation behavior 328 on the arrangement of the ssDNA suggests that the quadru329 plex structure is important in promoting LLPS with $\mathrm{H} 1$, re3 зо gardless of the kind of constituent nucleobases. In addition, ${ }_{331}$ we found that the droplet formation between quadruplex332 forming ssDNAs and polycationic chains such as $\mathrm{H} 1$ was ${ }_{3} 3$ synchronized with structural stabilization or transition.

\section{${ }_{34}$ Driving forces for the formation of droplets between ${ }_{35}$ DNAs and $\mathrm{H} 1$}

${ }_{336}$ Subsequently, we investigated the reason that the quadru${ }_{37}$ plex structures promote droplet formation between $\mathrm{H} 1$ and 338 SSDNA. To gain further insight into the driving forces of 339 droplet formation, we examined the effects of additives ${ }_{340}(\mathrm{NaCl}$ and 1,6-hexanediol) that can inhibit electrostatic and ${ }_{341}$ hydrophobic interactions, respectively, ${ }^{35}$ by adding them to 342 solutions containing the DNA/H1 liquid droplets. For com${ }_{343}$ parison, we also used 11-base pairs of double-stranded ${ }_{344}$ DNA (dsDNA11; Figure 1C), which had the same total num345 ber of nucleobases as the other ssDNAs. The duplex struc346 ture has some features similar to those of the quadruplex 347 structure: (i) nucleobases embedded by base pairing, (ii) a 348 relatively rigid structure, and (iii) LLPS when mixed with $349 \mathrm{H} 1.12,36$

(A)

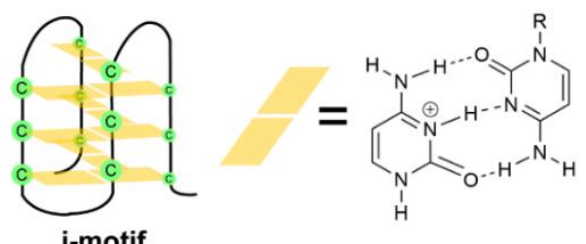

(B)
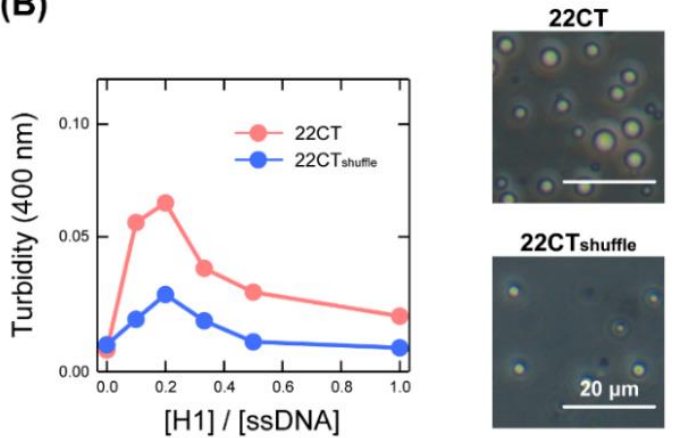

350

351 Figure 4. Droplet formation in a solution that contains cytosine352 based quadruplex structures and H1. (A) Schematic illustration 353 of the i-motif structure and the DNA sequences used in this ex354 periment. (B) Formation of liquid droplets in solutions of i-mo355 tif DNA (22CT) or a shuffled sequence (22CTshuffle) with H1 (2 $356 \mu \mathrm{M}$ ); scale bar $=20 \mu \mathrm{m}$. 
$357 \mathrm{NaCl}$ markedly reduced both the turbidity of the solution 358 and the droplet size (Figures 5A and S5). In all DNAs, the359 droplets disappeared completely at higher-than-physiolog360 ical $\mathrm{NaCl}$ concentrations $(\sim 300 \mathrm{mM})$, as observed previ${ }_{361}$ ously. ${ }^{12}$ This result demonstrates that the electrostatic in362 teractions between the cationic C-terminus of $\mathrm{H} 1$ and the 363 anionic phosphate groups of DNAs are the dominant driving 364 force in the generation of LLPS.

365 Unexpectedly, the droplets consisting of the various G366 quadruplex-forming ssDNAs gradually dissolved as the 1,6367 hexanediol concentration was increased, whereas the tur368 bidity of the solutions containing droplets formed by 369 dsDNA 11 did not change at all, even in the presence of $20 \%$ 370 1,6-hexanediol (Figures 5B and S6). This result suggests 371 that hydrophobic interactions contribute substantially to 372 the stabilization of the droplets of G-quadruplex-forming 373 SsDNAs, but not those of dsDNA 11 . To better understand the 374 nature of these hydrophobic interactions, the relationship 375 between LLPS and the nucleotide-monophosphate (NMP) 376 DNA structural units was examined. Among the four NMPs, 377 only guanine monophosphate (GMP) caused droplet for378 mation in the presence of both $\mathrm{H} 1$ and high concentrations 379 of PEG, which is known to promote the generation of LLPS 380 (Figures 5C and S7). ${ }^{37}$

Guanine has the lowest energy of stacking interaction 32 with both aromatic amino acids ${ }^{38}$ and nucleobases ${ }^{39}$ among 383 the nucleobases. Considering the fact that $\mathrm{H} 1$ contains only 384 several aromatic amino acids (only two Phe and three Tyr 385 in 194 aa), more stable stacking of the guanine bases may 386 explain the results observed for the various NMPs, i.e., it is 38 plausible that the hydrophobic $\pi-\pi$ stacking of guanine ba388 ses is a significant factor for the LLPS of G-quadruplexes.

389 Taken together, (i) electrostatic interactions between the 390 DNAs and the intrinsically disordered region of $\mathrm{H} 1$ and (ii) $391 \pi-\pi$ stacking between quadruplex DNAs drove droplet for392 mation between G-quadruplex DNA and H1, unlike in the 393 electrostatically driven LLPS of duplex DNA and H1. In ad394 dition to electrostatic interactions, $\pi-\pi$ interactions are 395 known to be significant in protein phase separation to di396 rect the state of the assembly towards liquid-like droplets 397 rather than gel-like aggregates. ${ }^{44}$ Hydrogen-bonding inter398 actions between partially exposed guanine bases and lysine 399 residues $^{45}$ of H1, and cation- $\pi$ interactions, which stabilize 400 a wide variety of intracellular droplets, ${ }^{46,47}$ between the ${ }_{01}$ same pairs may also contribute to the stability of the ${ }_{02}$ DNA/H1 droplets. These considerations were also sup${ }_{403}$ ported by an experiment using ssDNA with shuffled vari404 ants of G-quadruplex-forming sequences (for details, see 405 Section 7 of the Supporting Information).

\section{Role of structural rigidity on the formation of droplets between DNAs and $\mathbf{H 1}$}

408 Although the key driving forces have been identified, the 409 effects of the structuring of ssDNA on the formation of drop410 lets are still puzzling. It is generally believed that biological
(A)

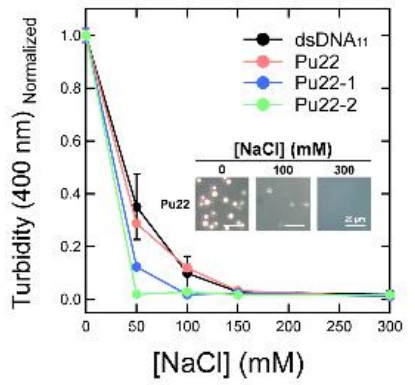

(C)

411

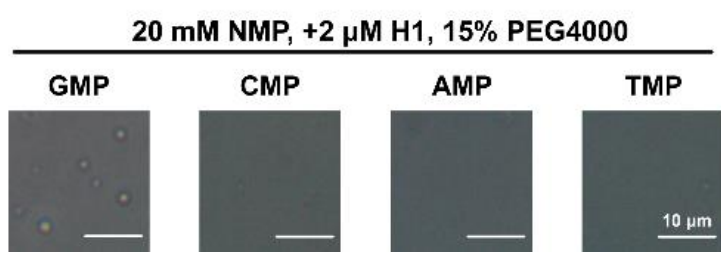

412 Figure 5. Physicochemical properties of the DNA/H1 liquid 413 droplets. (A and B) Turbidity of solutions of the ssDNA/H1 liq414 uid droplets in the presence of (A) $0-300 \mathrm{mM} \mathrm{NaCl}$ or (B) 0 $41520 \%$ 1,6-hexanediol. Insets are phase-contrast images of the ${ }_{416} \mathrm{Pu} 22 / \mathrm{H} 1$ liquid droplets in the presence of $\mathrm{NaCl}$; scale bar $=25$ $417 \mu \mathrm{m}$. (C) Phase-contrast microscopy images of solutions that 418 contain $20 \mathrm{mM} \mathrm{NMP,} 2 \mu \mathrm{M} \mathrm{H1}$, and 15\% PEG4000; scale bar = $41910 \mu \mathrm{m}$.

420 LLPS requires flexible sequences (e.g., IDR). ${ }^{48}$ It has been re${ }_{421}$ ported that when DNAs are stiffened by the formation of 422 secondary structures such as double strands or loops, they 423 form gel-like aggregates rather than liquid-like droplets in 424 the presence of cationic macromolecules, ${ }^{49,50}$ or are ex425 cluded from protein droplets. ${ }^{51}$ The high droplet formation 426 ability of the compact and rigid quadruplex folding struc427 ture seems to be inconsistent with these findings.

428 Therefore, the effect of structuring was investigated by 429 comparing the LLPS of flexible poly $(\mathrm{dA})$ and a highly rigid, 430 hydrophilic, spherical poly(amidoamine) (PAMAM) den${ }_{431}$ drimer with 64 carboxyl groups on its surface. According to 432 the phase diagrams of $\mathrm{H} 1$ vs. $\mathrm{NaCl}$ concentration (Figures 6 , ${ }_{433} \mathrm{~S} 8$ and S9), Pu22, whose rigidity is intermediate between 434 those of dsDNA ${ }^{40}$ and the PAMAM dendrimer, ${ }^{52}$ clearly ex435 hibited the highest phase separation ability (Figure 6). This 436 result implies that structural flexibility is not necessarily an 437 essential factor for LLPS with H1. The PAMAM dendrimer 438 can also be considered to be a model of polyanionic proteins, ${ }_{439}$ such as the histone chaperone prothymosin alpha, which 440 can bind and condense H1 without LLPS, 53 and our result 441 may be similar to the behavior of such proteins.

442 The observed high LLPS ability of quadruplex folding ${ }_{443}$ structures is possibly due to the formation of interfaces con${ }_{444}$ sisting of organized top/bottom planes of guanine bases 445 and the side surfaces with high charge density. As recently ${ }_{446}$ reported, interactions between interfaces formed by struc${ }_{447}$ turing can promote LLPS. ${ }^{44}$ Short dsDNAs also undergo 

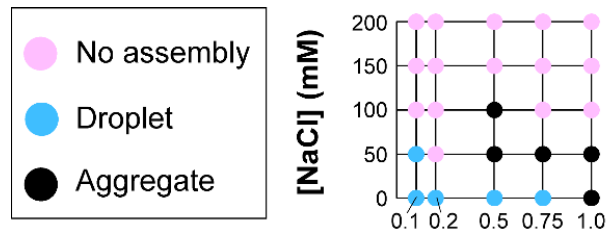

[H1] / [poly(dA)]

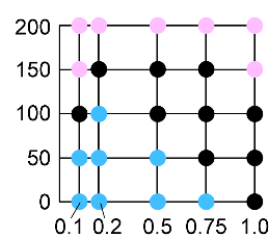

[H1] / [dsDNA 11$]$
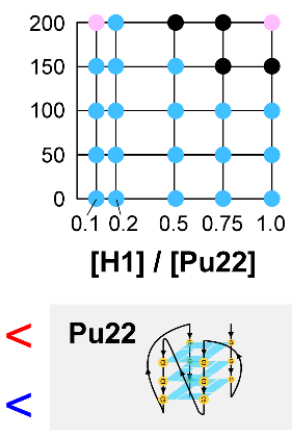

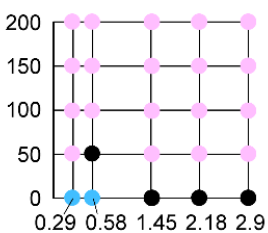

[H1] / [PAMAM]

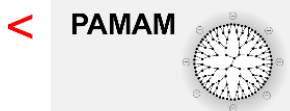

Figure 6. Phase diagrams of aqueous mixtures of $\mathrm{H} 1$ and various anionic molecules. The solution states are plotted as the NaCl concentration vs. the molar mixing ratio of $\mathrm{H} 1$ to DNAs $(10 \mu \mathrm{M})$ or PAMAM $(3.44 \mu \mathrm{M})$. The concentration range of PAMAM (64 anions per molecule) was chosen to have the same charge as the DNA solution (22 anions per molecule). Pink dots: no phase separation; blue dots: liquid-liquid phase separation; black dots: solid-liquid phase separation (i.e., aggregation). The order of the rigidity and charge density of the molecules was based on references 40 and 41, and 42 and 43, respectively.

454 hierarchical self-assembly by end-to-end stacking of base 455 pairs at the terminal interfaces upon droplet formation with 456 polycations. ${ }^{55}$ The G-quadruplex exhibits more open and 457 wider stacking interfaces compared to the duplex, and 45 DNAs with quadruplex structures tend to stack intermolec459 ularly in the longitudinal direction at high concentra460 tions. ${ }^{56,57}$ These characteristics explain the smaller contri461 bution of hydrophobic interactions in the duplexes com462 pared to that in the G-quadruplexes. Indeed, the phase dia${ }_{463}$ grams showed that $\mathrm{Pu} 22$ droplets appeared to be more tol${ }_{464}$ erant to salts than dsDNA 11 droplets (Figures 6 and S10). 465 This difference should be attributed to improved stability ${ }_{466}$ due to hydrophobic inter-nucleobase stackings provided by 467 the upper and lower surfaces of G-quartets. Turbidity meas468 urements (Figure 2A) showed that one H1 molecule bound 469 to multiple ssDNAs. This bridging of ssDNAs by H1 likely 40 contributed to the facilitation of the stacking, and eventually ${ }_{471}$ stabilized the intermolecular G-quadruplex formation.

The correlation between the LLPS ability of DNAs with ${ }_{73}$ the same number of charges [Pu22, dsDNA 11 , and poly $\left.(\mathrm{dA})\right]$ 44 and charge density (Figure 6) also suggests the importance 45 of increasing the charge density via structuring. In addition 476 to the planar interfaces preferred for $\pi-\pi$ stacking on the 477 top and bottom surfaces, the formation of high-charge-den478 sity interfaces on the side surfaces that allow strong electro479 static contacts ${ }^{58}$ provides unique features favorable for ${ }_{480}$ droplet formation. We thus concluded that the densification 481 of the electrostatic and stacking interactions via the for482 mation of such structures is a key to the high LLPS ability of 483 quadruplex folding structures.

484 The possible existence of G-quadruplex droplets in the 485 cell nucleus is suggested by the fact that the droplets formed 486 by G-quadruplexes became larger with increasing molecu487 lar concentration, even at physiological ionic strength, un488 like random structured DNA (Figure S11). The quadru489 plexes in the nucleus are inhomogeneously distributed de490 pending on the surrounding environment and protein
491 binding. For example, G-quadruplex DNAs and RNAs are 492 concentrated in the promoter and telomere regions of spe493 cific genes to form G-quadruplex clusters. ${ }^{14,15}$ The concen494 trations of G-quadruplex DNAs and RNAs thus formed in the 495 nucleus could potentially exceed the thresholds of LLPS lo496 cally and transiently, and could be involved, for example, in 497 H1-mediated chromatin LLPS that changes over time. ${ }^{36}$ 498 Careful observations of quadruplex DNAs in cells from the 499 perspective of LLPS will shed new light on the role of quad500 ruplex DNAs.

\section{${ }_{501}$ Conclusion}

502 In summary, we have demonstrated that the formation of 503 quadruplex structures in single-strand DNA (ssDNA), in504 cluding guanine-based parallel and anti-parallel G-quadru505 plexes and cytosine-based i-motif structures, promotes the 506 formation of liquid-like droplets with linker histone H1 via 507 liquid-liquid phase separation (LLPS). The quadruplex fold508 ing is maintained or, in some cases, promoted inside the 509 droplet. Increasing the quadruplex content decreases both 510 the motility and the density of the molecules that comprise 511 the droplet. These droplets are likely formed via not only 512 electrostatic interactions between the anionic ssDNA and 513 the cationic $\mathrm{C}$-terminus of $\mathrm{H} 1$, but also via $\pi-\pi$ interactions 514 between the quadruplex structures. Thus, DNA quadruplex 515 structures may be capable of regulating LLPS-mediated dy516 namic chromatin condensation in the nucleus. DNA and 517 RNA with G-quadruplex structures can selectively interact 518 with nuclear proteins such as fused in sarcoma (FUS) and 519 hnRNPA1, which cause amyotrophic lateral sclerosis (ALS) 520 and tend to phase-separate. ${ }^{59,60}$ Accordingly, we expect that 521 the quadruplex structure can act as a hub that regulates bi522 ological processes such as chromatin condensation in the 523 nucleus via LLPS.

\section{${ }_{524}$ AUTHOR INFORMATION}

${ }_{525}$ Corresponding Authors 
${ }_{526}$ Shunsuke Tomita - ORCID: orcid.org/0000-0002-0586-6976; 527 E-mail: s.tomita@aist.go.jp

528 Ryoji Kurita - ORCID; orcid.org/0000-0001-5666-9561;

529 Email: r.kurita@aist.go.jp

30 Notes

${ }_{531}$ The authors declare no competing financial interest.

\section{${ }_{32}$ ACKNOWLEDGMENT}

533 We thank Prof. Daisuke Miyoshi (Konan University) for fruitful 4 discussions. This study was partially supported by a 5 DAICENTER project grant from the DBT (Govt. of India), a spe6 cial strategic grant from AIST (Japan), and JSPS KAKENHI 37 grants $18 \mathrm{H} 02383$ and $19 \mathrm{~K} 22377$.

\section{REFERENCES}

39 (1) Kouzarides, T. Chromatin Modifications and Their Function. Cell 2007, 128 (4), 693-705.

(2) Criscione, S. W.; Teo, Y. V.; Neretti, N. The Chromatin Landscape of Cellular Senescence. Trends Genet. 2016, 32 (11), 751-761.

4 (3) Grunstein, M. Histone Acetylation in Chromatin Structure and Transcription. Nature 1997, 389 (6649), 349-352.

6 (4) Erdel, F.; Rippe, K. Formation of Chromatin Subcompartments by Phase Separation. Biophys. J. 2018, 114 (10), 2262-2270.

(5) Banani, S. F.; Lee, H. O.; Hyman, A. A.; Rosen, M. K. Biomolecular Condensates: Organizers of Cellular Biochemistry. Nat. Rev. Mol. Cell Biol. 2017, 18 (5), 285-298.

(6) Boeynaems, S.; Alberti, S.; Fawzi, N. L.; Mittag, T.; Polymenidou, M.; Rousseau, F.; Schymkowitz, J.; Shorter, J.; Wolozin, B.; Van Den Bosch, L.; Tompa, P.; Fuxreiter, M. Protein Phase Separation: A New Phase in Cell Biology. Trends Cell Biol. 2018, 28 (6), 420-435.

(7) Iwashita, K.; Mimura, M.; Shiraki, K. Control of Aggregation, Coaggregation, and Liquid Droplet of Proteins Using Small Additives. Curr. Pharm. Biotechnol. 2018, 19 (12), 946-955.

(8) Larson, A. G.; Elnatan, D.; Keenen, M. M.; Trnka, M. J.; Johnston, J. B.; Burlingame, A. L.; Agard, D. A.; Redding, S.; Narlikar, G. J. Liquid Droplet Formation by HP1 $\alpha$ Suggests a Role for Phase Separation in Heterochromatin. Nature 2017 547 (7662), 236-240.

(9) Strom, A. R.; Emelyanov, A. V.; Mir, M.; Fyodorov, D. V.; Darzacq, X.; Karpen, G. H. Phase Separation Drives Heterochromatin Domain Formation. Nature 2017, 547 (7662), 241-245.

(10) Gibson, B. A.; Doolittle, L. K.; Schneider, M. W. G.; Jensen, L. E.; Gamarra, N.; Henry, L.; Gerlich, D. W.; Redding, S.; Rosen, M. K. Organization of Chromatin by Intrinsic and Regulated Phase Separation. Cell 2019, 179 (2), 470-484.e21.

(11) Shin, Y.; Chang, Y.-C.; Lee, D. S. W.; Berry, J.; Sanders, D. W.; Ronceray, P.; Wingreen, N. S.; Haataja, M.; Brangwynne, C. P. Liquid Nuclear Condensates Mechanically Sense and Restructure the Genome. Cell 2018, 175 (6), 1481-1491.e13.

(12) Turner, A. L.; Watson, M.; Wilkins, O. G.; Cato, L.; Travers, A.; Thomas, J. 0.; Stott, K. Highly Disordered Histone H1- DNA Model Complexes and Their Condensates. Proc. Natl. Acad. Sci. U. S. A. 2018, 115 (47), 11964-11969.

(13) Burge, S.; Parkinson, G. N.; Hazel, P.; Todd, A. K.; Neidle, S. Quadruplex DNA: Sequence, Topology and Structure. Nucleic Acids Res. 2006, 34 (19), 5402-5415.

4 (14) Yoshida, W.; Saikyo, H.; Nakabayashi, K.; Yoshioka, H.; Bay, D. H.; Iida, K.; Kawai, T.; Hata, K.; Ikebukuro, K.; Nagasawa,
K.; Karube, I. Identification of G-Quadruplex Clusters by High-Throughput Sequencing of Whole-Genome Amplified Products with a G-Quadruplex Ligand. Sci. Rep. 2018, 8 (1), 3116.

15) Xu, Y.; Suzuki, Y.; Ito, K.; Komiyama, M. Telomeric RepeatContaining RNA Structure in Living Cells. Proc. Natl. Acad. Sci. U. S. A. 2010, 107 (33), 14579-14584.

16) Murat, P.; Balasubramanian, S. Existence and Consequences of G-Quadruplex Structures in DNA. Curr. Opin. Genet. Dev. 2014, 25 (1), 22-29.

17) Paeschke, K.; Simonsson, T.; Postberg, J.; Rhodes, D.; Lipps, H. J. Telomere End-Binding Proteins Control the Formation of G-Quadruplex DNA Structures in Vivo. Nat. Struct. Mol. Biol. 2005, 12 (10), 847-854.

8) Hoffmann, R. F.; Moshkin, Y. M.; Mouton, S.; Grzeschik, N. A.; Kalicharan, R. D.; Kuipers, J.; Wolters, A. H. G.; Nishida, K.; Romashchenko, A. V.; Postberg, J.; Lipps, H.; Berezikov, E.; Sibon, O. C. M.; Giepmans, B. N. G.; Lansdorp, P. M. Guanine Quadruplex Structures Localize to Heterochromatin. Nucleic Acids Res. 2016, 44 (1), 152-163.

9) Perišić, O.; Schlick, T. Dependence of the Linker Histone and Chromatin Condensation on the Nucleosome Environment. J. Phys. Chem. B 2017, 121 (33), 7823-7832.

Shakya, A.; King, J. T. Non-Fickian Molecular Transport in Protein-DNA Droplets. ACS Macro Lett. 2018, 7 (10), 12201225.

1) Ambrus, A.; Chen, D.; Dai, J.; Jones, R. A.; Yang, D. Solution Structure of the Biologically Relevant G-Quadruplex Element in the Human c-MYC Promoter. Implications for GQuadruplex Stabilization. Biochemistry 2005, 44 (6), 20482058.

Ishida, T.; Kinoshita, K. PrDOS: Prediction of Disordered Protein Regions from Amino Acid Sequence. Nucleic Acids Res. 2007, 35, W460-464.

(23) Kurinomaru, T.; Maruyama, T.; Izaki, S.; Handa, K.; Kimoto, T.; Shiraki, K. Protein-Poly(Amino Acid) Complex Precipitation for High-Concentration Protein Formulation. $J$. Pharm. Sci. 2014, 103 (8), 2248-2254.

24) Matsuda, A.; Mimura, M.; Maruyama, T.; Kurinomaru, T.; Shiuhei, M.; Shiraki, K. Liquid Droplet of ProteinPolyelectrolyte Complex for High-Concentration Formulations. J. Pharm. Sci. 2018, 107 (10), 2713-2719.

(25) Mimura, M.; Tsumura, K.; Matsuda, A.; Akatsuka, N.; Shiraki, K. Effect of Additives on Liquid Droplet of ProteinPolyelectrolyte Complex for High-Concentration Formulations. J. Chem. Phys. 2019, 150 (6), 064903.

26) Brangwynne, C. P.; Eckmann, C. R.; Courson, D. S.; Rybarska, A.; Hoege, C.; Gharakhani, J.; Jülicher, F.; Hyman, A. A Germline P Granules Are Liquid Droplets That Localize by Controlled Dissolution/Condensation. Science 2009, 324 (5935), 1729-1732.

(27) Taylor, N. O.; Wei, M.-T.; Stone, H. A.; Brangwynne, C. P. Quantifying Dynamics in Phase-Separated Condensates Using Fluorescence Recovery after Photobleaching. Biophys. J. 2019, 117 (7), 1285-1300.

(28) Gabelica, V.; Maeda, R.; Fujimoto, T.; Yaku, H.; Murashima, T. Sugimoto, N.; Miyoshi, D. Multiple and Cooperative Binding of Fluorescence Light-up Probe Thioflavin T with Human Telomere DNA G-Quadruplex. Biochemistry 2013, 52 (33), 5620-5628.

29) Nicoludis, J. M.; Barrett, S. P.; Mergny, J.-L.; Yatsunyk, L. A. Interaction of Human Telomeric DNA with N-Methyl Mesoporphyrin IX. Nucleic Acids Res. 2012, 40 (12), 54325447. 
650 (30) Lin, Y.; Protter, D. S. W.; Rosen, M. K.; Parker, R. Formation and Maturation of Phase-Separated Liquid Droplets by RNABinding Proteins. Mol. Cell 2015, 60 (2), 208-219.

(31) Simpson, A. J.; Kingery, W. L.; Shaw, D. R.; Spraul, M.; Humpfer, E.; Dvortsak, P. The Application of ${ }^{1} \mathrm{H}$ HR-MAS NMR Spectroscopy for the Study of Structures and Associations of Organic Components at the Solid- Aqueous Interface of a Whole Soil. Environ. Sci. Technol. 2001, 35 (16), 3321-3325.

(32) Miyoshi, D.; Karimata, H.; Sugimoto, N. Hydration Regulates Thermodynamics of G-Quadruplex Formation under Molecular Crowding Conditions. J. Am. Chem. Soc. 2006, 128 (24), 7957-7963.

(33) Xue, Y.; Kan, Z.-Y.; Wang, Q.; Yao, Y.; Liu, J.; Hao, Y.-H.; Tan, Z Human Telomeric DNA Forms Parallel-Stranded Intramolecular G-Quadruplex in $\mathrm{K}+$ Solution under Molecular Crowding Condition. J. Am. Chem. Soc. 2007, 129 (36), 11185-11191.

(34) Phan, A. T.; Mergny, J. Human Telomeric DNA: G quadruplex, I - motif and Watson-Crick Double Helix. Nucleic Acids Res. 2002, 30 (21), 4618-4625.

(35) Lin, Y.; Mori, E.; Kato, M.; Xiang, S.; Wu, L.; Kwon, I.; McKnight, S. L. Toxic PR Poly-Dipeptides Encoded by the C9orf72 Repeat Expansion Target LC Domain Polymers. Cell 2016 167 (3), 789-802.e12.

(36) Shakya, A.; Park, S.; Rana, N.; King, J. T. Liquid-Liquid Phase Separation of Histone Proteins in Cells: Role in Chromatin Organization. Biophys. J. 2020, 118 (3), 753-764.

(37) Park, S.; Barnes, R.; Lin, Y.; Jeon, B.-J.; Najafi, S.; Delaney, K. T.; Fredrickson, G. H.; Shea, J.-E.; Hwang, D. S.; Han, S. Dehydration Entropy Drives Liquid-Liquid Phase Separation by Molecular Crowding. Commun. Chem. 2020, 3 (1), 83.

(38) Rutledge, L. R.; Campbell-Verduyn, L. S.; Wetmore, S. D. Characterization of the Stacking Interactions between DNA or RNA Nucleobases and the Aromatic Amino Acids. Chem. Phys. Lett. 2007, 444 (1), 167-175.

(39) Hobza, P.; Šponer, J. Structure, Energetics, and Dynamics of the Nucleic Acid Base Pairs: Nonempirical Ab Initio Calculations. Chem. Rev. 1999, 99 (11), 3247-3276.

(40) Cohen, H.; Sapir, T.; Borovok, N.; Molotsky, T.; Di Felice, R.; Kotlyar, A. B.; Porath, D. Polarizability of G4-DNA Observed by Electrostatic Force Microscopy Measurements. Nano Lett. 2007, 7 (4), 981-986.

(41) Sim, A. Y. L. Nucleic Acid Polymeric Properties and Electrostatics: Directly Comparing Theory and Simulation with Experiment. Adv. Colloid Interface Sci. 2016, 232, 4956.

(42) Zhang, Y.; Zhou, H.; Ou-Yang, Z. -C. Stretching SingleStranded DNA: Interplay of Electrostatic, Base-Pairing, and Base-Pair Stacking Interactions. Biophys. J. 2001, 81 (2), 1133-1143.

(43) Gatto, B.; Palumbo, M.; Sissi, C. Nucleic Acid Aptamers Based on the G-Quadruplex Structure: Therapeutic and Diagnostic Potential. Curr. Med. Chem. 2009, 16 (10), 1248-1265.

(44) Vernon, R. M.; Chong, P. A.; Tsang, B.; Kim, T. H.; Bah, A. Farber, P.; Lin, H.; Forman-Kay, J. D. Pi-Pi Contacts Are an
Overlooked Protein Feature Relevant to Phase Separation. Elife 2018, 7, e31486.

Czyżnikowska, Ż.; Lipkowski, P.; Góra, R. W.; Zaleśny, R.; Cheng, A. C. On the Nature of Intermolecular Interactions in Nucleic Acid Base-Amino Acid Side-Chain Complexes. J. Phys. Chem. B 2009, 113 (33), 11511-11520.

Alshareedah, I.; Kaur, T.; Ngo, J.; Seppala, H.; Kounatse, L.-A. D.; Wang, W.; Moosa, M. M.; Banerjee, P. R. Interplay between Short-Range Attraction and Long-Range Repulsion Controls Reentrant Liquid Condensation of Ribonucleoprotein-RNA Complexes. J. Am. Chem. Soc. 2019, 141 (37), 14593-14602.

Lin, Y.-H.; Forman-Kay, J. D.; Chan, H. S. Sequence-Specific Polyampholyte Phase Separation in Membraneless Organelles. Phys. Rev. Lett. 2016, 117 (17), 178101.

8) Uversky, V. N. Intrinsically Disordered Proteins in Overcrowded Milieu: Membrane-Less Organelles, Phase Separation, and Intrinsic Disorder. Curr. Opin. Struct. Biol. 2017, 44, 18-30.

49) Shakya, A.; King, J. T. DNA Local-Flexibility-Dependent Assembly of Phase-Separated Liquid Droplets. Biophys. J. 2018, 115 (10), 1840-1847.

V) Vieregg, J. R.; Lueckheide, M.; Marciel, A. B.; Leon, L.; Bologna, A. J.; Rivera, J. R.; Tirrell, M. V. Oligonucleotide-Peptide Complexes: Phase Control by Hybridization. J. Am. Chem. Soc. 2018, 140 (5), 1632-1638.

1) Nott, T. J.; Craggs, T. D.; Baldwin, A. J. Membraneless Organelles Can Melt Nucleic Acid Duplexes and Act as Biomolecular Filters. Nat. Chem. 2016, 8 (6), 569-575.

Chen, Z.; Chen, L.; Ma, H.; Zhou, T.; Li, X. Aptamer Biosensor for Label-Free Impedance Spectroscopy Detection of Potassium Ion Based on DNA G-Quadruplex Conformation. Biosens. Bioelectron. 2013, 48, 108-112.

53) George, E. M.; Brown, D. T. Prothymosin Alpha Is a Component of a Linker Histone Chaperone. FEBS Lett. 2010 , 584 (13), 2833-2836.

(54) Conicella, A. E.; Dignon, G. L.; Zerze, G. H.; Schmidt, H. B.; D’Ordine, A. M.; Kim, Y. C.; Rohatgi, R.; Ayala, Y. M.; Mittal, J.; Fawzi, N. L. TDP-43 $\alpha$-Helical Structure Tunes Liquid-Liquid Phase Separation and Function. Proc. Natl. Acad. Sci. U. S. A. 2020, 117 (11), 5883-5894.

5) Fraccia, T. P.; Jia, T. Z. Liquid Crystal Coacervates Composed of Short Double-Stranded DNA and Cationic Peptides. ACS Nano 2020, 14 (11), 15071-15082.

6) Guittet, E.; Renciuk, D.; Leroy, J.-L. Junctions between I-Motif Tetramers in Supramolecular Structures. Nucleic Acids Res. 2012, 40 (11), 5162-5170.

(57) Vorlíčková, M.; Kejnovská, I.; Sagi, J.; Renčiuk, D.; Bednářová, K.; Motlová, J.; Kypr, J. Circular Dichroism and Guanine Quadruplexes. Methods 2012, 57 (1), 64-75.

58) Alberti, P.; Mergny, J.-L. DNA Duplex-Quadruplex Exchange as the Basis for a Nanomolecular Machine. Proc. Natl. Acad. Sci. U. S. A. 2003, 100 (4), 1569-1573.

59) Zhang, Q.-S.; Manche, L.; Xu, R.-M.; Krainer, A. R. HnRNP A1 Associates with Telomere Ends and Stimulates Telomerase Activity. RNA 2006, 12 (6), 1116-1128.

Takahama, K.; Takada, A.; Tada, S.; Shimizu, M.; Sayama, K.; Kurokawa, R.; Oyoshi, T. Regulation of Telomere Length by G-Quadruplex Telomere DNA- and TERRA-Binding Protein TLS/FUS. Chem. Biol. 2013, 20 (3), 341-350. 


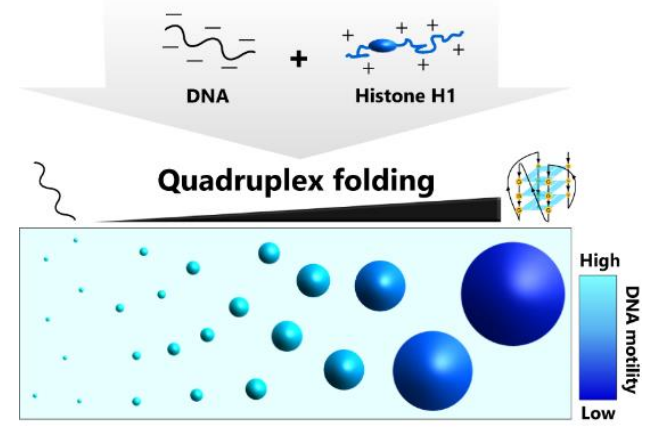

